\title{
Lo stato di qualità dei corpi idrici superficiali: classificazione ed analisi dei trend degli elementi LIMeco per Mingardo, Tusciano e Regi Lagni
}

\author{
Davide Comarco ${ }^{1}$
}

${ }^{1}$ Affiliation not available

\begin{abstract}
Lo scopo del presente elaborato è analizzare i trend dei parametri chimico-fisici che rientrano nella classificazione di un fiume. Questi, abbinati ad altri parametri concorrono alla determinazione finale dello Stato ecologico.

Per la classificazione dello stato ecologico dei corpi idrici fluviali gli elementi fisico-chimici a sostegno del biologico da utilizzare sono costituiti dai nutrienti di cui fanno parte l'azoto ammoniacale, l'azoto nitrico ed il fosforo totale oltre all'ossigeno disciolto.

Rispetto a tali elementi sono stati creati dei grafici che raffigurassero l'andamento temporale e l'andamento spaziale dei vari fiumi, partendo dai primi campionamenti avvenuti all'inizio degli anni 2000.

L'obiettivo è quello di analizzare i monitoraggi avvenuti degli elementi sopra citati per avere un quadro efficace e chiaro dello stato di qualità dei corpi idrici superficiali, nel suddetto caso fiumi Campani ma l'analisi può e dev'essere estesa anche all'interno reticolo idrografico italiano.
\end{abstract}

\section{Introduzione}

L'acqua rappresenta il settore con la maggiore copertura a livello normativo. La direttiva quadro sulle acque definisce un approccio comune a livello europeo per la gestione e la salvaguardia di 
tale bene. La direttiva stabilisce gli obiettivi per le condizioni dell'acqua in Europa, comprendendo acque sotterranee, superficiali e marino-costiere ${ }^{1}$.

L'obiettivo per la normativa è il raggiungimento dello stato "buono" sia chimico che ecologico entro il 21 dicembre 2015 e conseguentemente il mantenimento di tale stato, per i fiumi che non dovessero verificare lo stato sopra citato, l'obiettivo è quello di evitare ulteriori deterioramenti ${ }^{2}$.

Lo strumento principale utilizzato è la suddivisione in bacini idrografici del territorio, che attraverso l'ente denominato Autorità di bacino monitorerà l'andamento dei parametri dei fiumi all'interno del bacino di competenza ${ }^{3}$.

Per uniformare l'azione sono redatti a livello di aree territoriali specifiche dei piani di gestione con valenza sessennale, il primo redatto nel 2010, con lo scopo di attuare un'azione comune in materia di acque.

Con il Decreto Legislativo n.152/2006 ed in particolare l'art.64 l'Italia è suddivisa in 8 distretti idrografici e prevede per ogni distretto la redazione di un piano di gestione, attribuendone la competenza all' Autorità di distretto idrografico ${ }^{4}$.

La Campania assieme a Puglia, Basilicata, Calabria e parte del Lazio e del Molise costituisce il distretto idrografico dell' Appenino meridionale.

In tale studio approfondiremo la classificazione dei corpi idrici fluviali Campani quali Mingardo, Tusciano e Regi Lagni oltre ad analizzare l'andamento degli elementi costituenti l'indicatore LIMeco, che ricordiamo essere azoto ammoniacale, azoto nitrico, fosforo totale ed ossigeno disciolto. 


\section{Analisi dei parametri}

Come anticipato precedentemente sono stati analizzati tre fiumi Campani di differente qualità ambientale ${ }^{3}$.

Come primo fiume è stato analizzato il Mingardo, corpo idrico ubicato all'interno del bacino idrografico della sinistra Sele ${ }^{5}$.

L'analisi è stata condotta sugli elementi dell'indicatore LIMeco quindi azoto ammoniacale, azoto nitrico, fosforo totale e ossigeno disciolto ${ }^{6}$.

L'andamento di ogni singolo parametro non si discosta dai valori naturali ${ }^{7}$, essi infatti sono prossimi allo zero e comunque all'interno dei valori rappresentanti uno stato ecologico elevato-buono. Anche l'ossigeno disciolto presenta valori ottimali nell'intorno dei $10 \mathrm{mg} / \mathrm{L}$.

L'andamento spaziale non mostra valori in crescita spostandoci da monte verso valle ma medie pressoché costanti.

Secondo fiume analizzato è il Tusciano, fiume ubicato all' interno del bacino idrografico della destra Sele.

L'andamento temporale dei parametri mostra che il primo sito di campionamento indicato con la sigla Tu1 mostra valori accettabili ed all'interno di uno stato elevato-buono con valori raramente all'interno dello stato sufficiente ${ }^{8}$. Per tale fiume si nota un peggioramento sempre maggiore muovendoci verso la foce con le stazioni di monitoraggio denominate con la sigla Tu2 e Tu3, ciò probabilmente dovuto ad una attività antropica ed industriale diffusa. Tutto ciò appena detto è confermato dai grafici box plot che mostrano un aumento dei valori medi passando dal primo sito di campionamento all'ultimo ${ }^{5}$.

Discorso totalmente differente per l'ultimo fiume analizzato ovvero il Regi Lagni, quest'ultimo 
ubicato all'interno del bacino idrografico Nord-Occidentale della Campania9 .

Oltre all'ossigeno disciolto che, tuttavia, presenta valori accettabili seppur molto bassi come quantitativo di ossigeno presente all'interno della matrice acquosa e che difficilmente permette la salvaguardia della fauna ittica al suo interno, gli elementi azoto ammoniacale, azoto nitrico e fosforo totale presentano concentrazioni preoccupanti ${ }^{10}$.

Difatti, i valori sono ampiamenti al di fuori dei limiti imposti da normativa e questo sin dall'inizio dei primi campionamenti e già da monte le rilevazioni indicato valori molto elevati ${ }^{11}$.

Il fiume dai primi campionamenti e dalle prime classificazioni ha sempre verificato uno stato ecologico pessimo ed un altrettanto stato chimico ${ }^{12}$. Ciò desta particolare preoccupazione anche in relazione alla salute della popolazione che quotidianamente potrebbe approvvigionarsi di tale acqua ${ }^{6}$.

\section{Conclusioni}

In conclusione, è di fondamentale importanza mappare e classificare la totalità dei corpi idrici superficiali oltre a tutti i restanti tipi di acqua quale sotterranea e marino-costiera.

Avere una buona qualità delle acque significa avere anche una buona qualità della vita essendo quasi ogni nostro gesto o azione riconducibile all'utilizzo dell'acqua stessa.

Lo studio era incentrato sull'analisi di tre fiumi Campani ma questo può e dev'essere esteso alla totalità dei fiumi italiani ed europei per avere un quadro chiaro ed univoco dei corpi idrici fluviali ed intervenire ove sia necessario o, meglio ancora, evitare il raggiungimento di una qualità ambientale pessima come nel caso del Regi Lagni.

Gli sviluppi futuri possono riguardare sicuramente l'aumento del numero di stazioni di monitoraggio per avere un quadro sempre più preciso e puntuale dello stato di qualità di ogni singolo fiume. 


\section{References}

1.Kallis, G. The EU water framework directive: measures and implications. Water Policy 3, $125-142$ (2001).

2.Naddeo, V., Zarra, T. \& Belgiorno, V. Optimization of sampling frequency for river water quality assessment according to Italian implementation of the EU Water Framework Directive. Environmental Science \& Policy 10, 243-249 (2007).

3.Naddeo, V. \& Taherzadeh, M. J. Biomass valorization and bioenergy in the blue circular economy. Biomass and Bioenergy 149, 106069 (2021).

4.Corpuz, M. V. A. et al.. Wastewater treatment and fouling control in an electro algae-activated sludge membrane bioreactor. Science of The Total Environment 786, 147475 (2021).

5.Ibrahim, Y., Abdulkarem, E., Naddeo, V., Banat, F. \& Hasan, S. W. Synthesis of super hydrophilic cellulose-alpha zirconium phosphate ion exchange membrane via surface coating for the removal of heavy metals from wastewater. Science of The Total Environment 690, 167-180 (2019).

6.Lado, J. J. et al.. Performance analysis of a capacitive deionization stack for brackish water desalination. Desalination 501, 114912 (2021).

7.Senatore, V. et al. . Innovative membrane photobioreactor for sustainable CO2 capture and utilization. Chemosphere 273, 129682 (2021).

8.Prado, M. et al.. Removal of emerging contaminant and fouling control in membrane bioreactors by combined ozonation and sonolysis. International Biodeterioration \& Biodegradation 119, 577-586 (2017).

9.Fraiese, A. et al.. Ultrasonic processes for the advanced remediation of contaminated sediments. Ultrasonics Sonochemistry 67, 105171 (2020). 
10.Ali, A. A. A., Naddeo, V., Hasan, S. W. \& Yousef, A. F. Correlation between bacterial community structure and performance efficiency of a full-scale wastewater treatment plant. Journal of Water Process Engineering 37, 101472 (2020).

11.Jallouli, S. et al.. Efficient and sustainable treatment of tannery wastewater by a sequential electrocoagulation-UV photolytic process. Journal of Water Process Engineering 38, 101642 (2020).

12.Naddeo, V. \& Korshin, G. Water energy and waste: The great European deal for the environment. Science of The Total Environment 764, 142911 (2021).

13.Ibrahim, Y., Abdulkarem, E., Naddeo, V., Banat, F. \& Hasan, S. W. Synthesis of super hydrophilic cellulose-alpha zirconium phosphate ion exchange membrane via surface coating for the removal of heavy metals from wastewater.. Sci Total Environ 690, 167-180 (2019).

14.Borea, L. et al.. Wastewater treatment by membrane ultrafiltration enhanced with ultrasound: Effect of membrane flux and ultrasonic frequency. Ultrasonics 83, 42-47 (2018).

15.Ardakani, M. N. \& Gholikandi, G. B. Microbial fuel cells (MFCs) in integration with anaerobic treatment processes (AnTPs) and membrane bioreactors (MBRs) for simultaneous efficient wastewater/sludge treatment and energy recovery -A state-of-the-art review. Biomass and Bioenergy 141, 105726 (2020). 


\section{Figure Captions}

Figure 1. Corpo idrico fluviale in fase di monitoraggio.

Figure 2. Andamento spaziale dell'ossigeno disciolto per il Mingardo. 
Figures

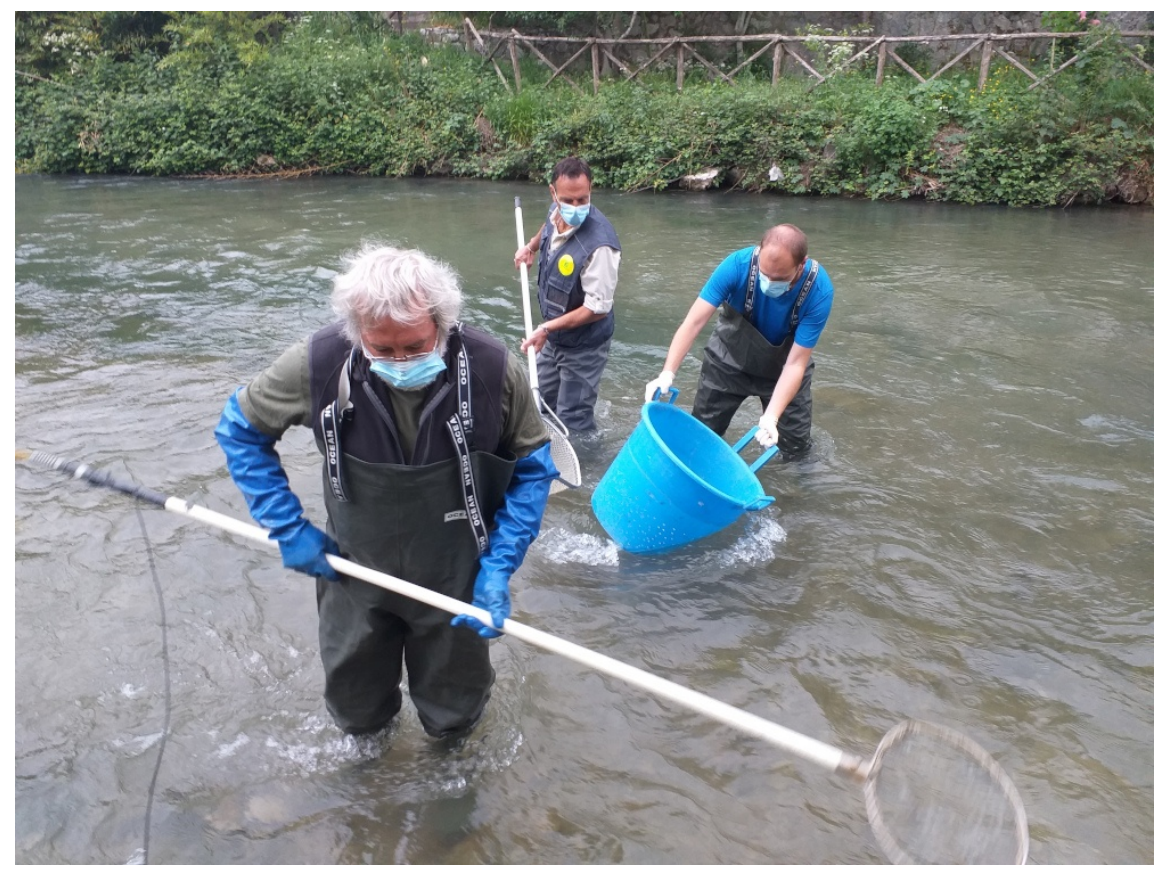

Figure 1: Corpo idrico fluviale in fase di monitoraggio. 


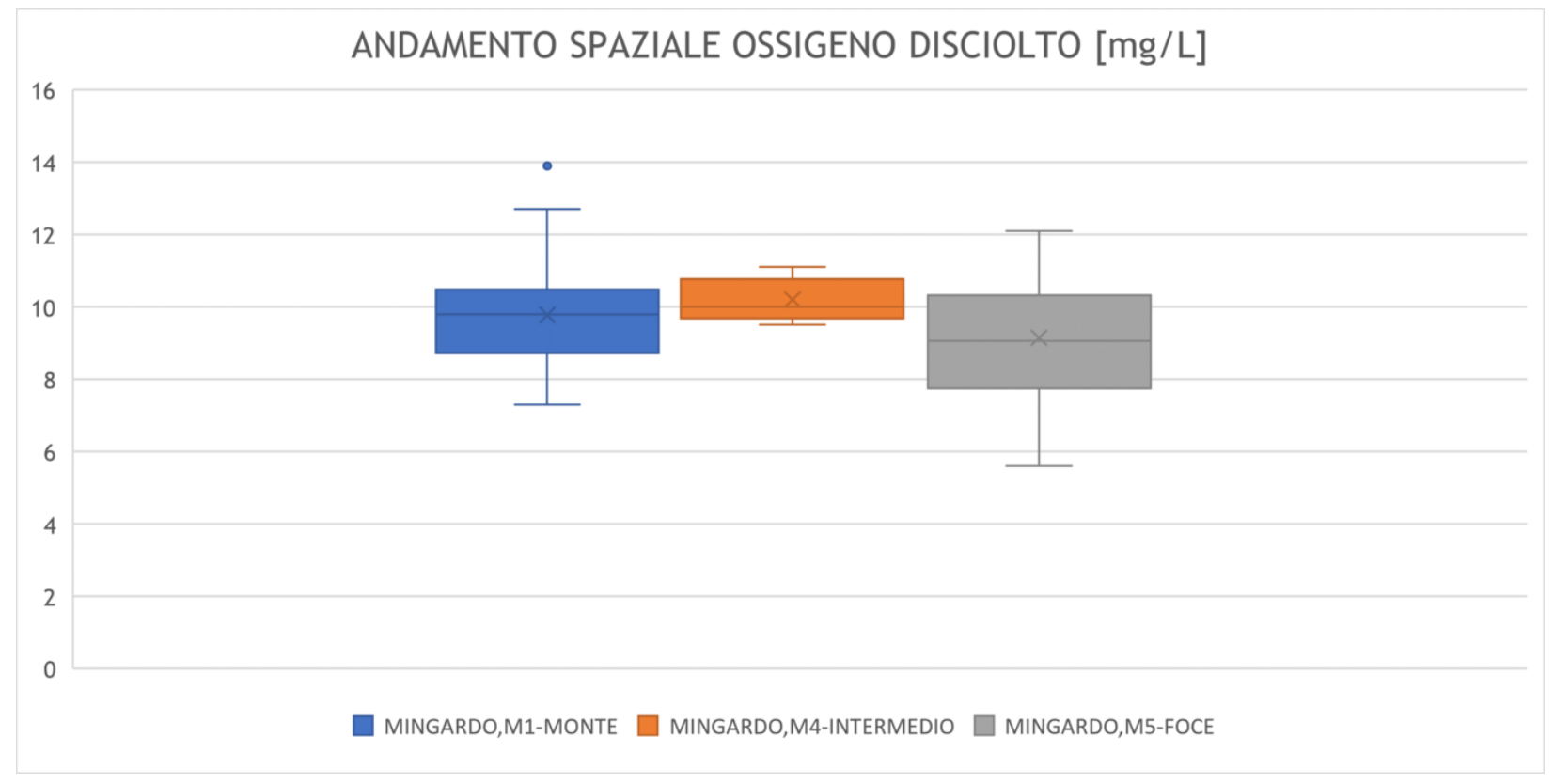

Figure 2: Andamento spaziale dell'ossigeno disciolto per il Mingardo. 